\title{
Uptake of Breast and Cervical Cancer Screening in Four Gulf Cooperation
}

\section{Countries}

\section{Short Title: Uptake of Cancer Screening in GCC Countries}

Vivian H.T. So ${ }^{1}$, Andrew Amos Channon ${ }^{2 *}$, Mohamed M. Ali ${ }^{3}$, Leena Merdad ${ }^{4}$, Sultana Al

Sabahi $^{5}$, Huda Al Suwaidi ${ }^{6}$, Abdullah Al Ajeel $^{7}$, Nabil Osman $^{8}$, Tawfik A M Khoja $^{9}$

* Corresponding Author

${ }^{1} \mathrm{PhD}$, Faculty of Health Sciences and Sport, University of Stirling, UK, Vivian.so@stir.ac.uk

${ }^{2} \mathrm{PhD}$, Department of Social Statistics and Demography, University of Southampton, UK, A.R.

\section{Channon@soton.ac.uk}

${ }^{3}$ PhD, World Health Organisation, Geneva, alim@who.int

${ }^{4}$ Doctorate of Science (SD), King Abdulaziz University (Department of Dental Public Health, Faculty of Dentistry), Jeddah, Saudi Arabia, lam641@mail.harvard.edu

${ }^{5} \mathrm{MSc}$, Center of Studies and Research, Ministry of Health, Muscat, Oman, al-

\section{sabahis@hotmail.com}

${ }^{6}$ Arab Board Family Medicine, PhD, Vulnerable Group Department, Community

Development Authority, Dubai, UAE, alsuwaidi.huda71@gmail.com

${ }^{7}$ MD Public Health, Health \& Vital Statistics, National Center of Health Information, Ministry

of Health, Kuwait, aalajeel@moh.gov.kw

${ }^{8}$ BSc, Ministry of Health, Saudi Arabia, abushadi@moh.gov.sa 
${ }^{9}$ MBBS, DPHC, FRCGP, FFPH, FRCP, School of Public Health, Primary Care \& Public Health, Imperial College, London, UK, khojatawfik@gmail.com

\section{Source of Funding}

This paper is financially supported by the World Health Organisation Regional Office of Eastern Mediterranean, Cairo, Egypt.

\section{Conflicts of Interest}

None declared 


\begin{abstract}

\section{Objectives}

In Gulf Cooperation Council (GCC) States there is limited information on national levels of mammography and Pap smear screening uptake. The aim of this study is to provide a baseline for national estimates for mammography and Pap smear screening and to explore associations between screening uptake and socio-economic factors.
\end{abstract}

\title{
Methods
}

The nationally representative World Health Survey+, implemented in 2008/9 in Kuwait, Oman, Saudi Arabia and United Arab Emirates, was used. Uptake of Mammography and Pap smear were estimated for each country, followed by the examination of associations between screening and a range of socio-economic variables.

\section{Results}

Levels of breast and cervical cancer screening uptake within recommended intervals in all countries were low. The percentages of women aged 40-75 who had a mammogram were 4.9\% in Saudi Arabia, $8.9 \%$ in Oman, $13.9 \%$ in the UAE and $14.6 \%$ in Kuwait. The percentages of women aged 25-49 who had a Pap smear test were $7.6 \%$ in Saudi Arabia, $10.6 \%$ in Oman, $17.7 \%$ in Kuwait and $28.0 \%$ in the UAE. Marital status, wealth, education, nationality and place of residence are associated with screening uptake, with the lower educated, poor and unmarried having the lowest percentages of uptake.

\section{Conclusions}


The four GCC countries need to set clear targets and increase the proportion of women who have regular breast and cervical cancer screening examinations. Health education campaigns and awareness programmes that are fully integrated into the health system are required to ensure women use services that are available to prevent breast and cervical cancers.

Keywords: Mass Screening; breast cancer; cervical cancer; Mammography; Papanicolaou test; health surveys; Gulf Cooperation Countries 


\section{Introduction}

In 2012, approximately 1.67 million new cases of breast cancer and 528,000 cases of cervical cancer were recorded worldwide (Ferlay et al., 2015). In the Gulf Cooperation Council (GCC) region breast cancer ranked first (24.2\% of total diagnoses) and cervical cancer ranked seventh (2.9\% of total diagnoses) for cancer incidence amongst females between 1998 and 2009 (Al-Othman et al., 2015). It has been estimated that there were 42,000 deaths due to breast cancer (13.9 deaths / 100,000 women) and 8,000 deaths due to cervical cancer (2.6 deaths / 100,000 women) in the WHO Eastern Mediterranean Region in 2012 (Ervik et al., 2016). There is evidence that in GCC countries both breast and cervical cancers are diagnosed later and affect younger groups than seen elsewhere (Al-Othman et al., 2015). Screening for frequently observed cancer types, such as cervical and breast cancer, is effective in ensuring early diagnosis and appropriate treatment (World Health Organization, 2015). However, the scarce information about the use of screening programmes for breast and cervical cancer within the GCC region indicates that these services are underutilised (El Bcheraoui et al., 2015).

Mammography and Papanicolaou (Pap) smear screening are recommended as the most effective general screening tests for reducing mortality from breast and cervical cancer (World Health Organization, 2013, 2014). The WHO recommends mammogram screening with an interval of two years for those aged 50-69, with screening programmes for those aged 40-49 and 70-75 in well-resourced settings with appropriate research and monitoring and evaluation (World Health Organization, 2014). Due to the slow-growing nature of most cervical cancers, early detection is known to greatly reduce mortality (Al Eyd and Shaik, 2012). WHO recommends a cervical screening interval of every three years for women aged 
between 25 and 49 years old and a five-year interval for women aged 50 to 64 years old, if resources are available (World Health Organization, 2013).

Nationally representative information regarding screening for cancers in the GCC region is scarce. A nationally representative study on breast cancer screening from Saudi Arabia indicated very low levels of mammogram use amongst women aged over 50 years (EI Bcheraoui et al., 2015). Further studies, conducted in a number of GCC countries, have focused on specific population groups and show that screening use is low (Bener et al., 2009, Ravichandran et al., 2011, Donnelly et al., 2012, Latif, 2014).

There is a consensus that cancer awareness is a driving factors for women to engage in screening activities (Ravichandran et al., 2011, Donnelly et al., 2012, Sait et al., 2012, Donnelly et al., 2015a). This knowledge is influenced by sociodemographic and cultural factors, with younger, more educated, married and employed women more likely to be aware of and use the available screening (Ravichandran et al., 2011, Donnelly et al., 2015a). The household context is also important within the GCC region, with studies identifying the importance of husbands and male household members in women's participation in screening (Al-Amoudi and Abdulijabbar, 2012, Donnelly et al., 2015b).

The current literature on breast and cervical cancer screening in the GCC region mainly focuses on a single country with a resulting lack of comparative information. Research is mainly conducted at the subnational level amongst specific age groups. A comparative study will identify areas of good practice within the region as well as providing data that can be used as a baseline to assess the efficacy of policies to increase screening use. This paper examines the screening levels and the relationship between background factors and screening for both breast and cervical cancer in four GCC countries (Kuwait, Oman, Saudi Arabia and UAE) using nationally representative survey data. 


\section{Material and Methods}

\section{Data}

The World Health Survey Plus (WHS+) was conducted in Kuwait (2008), Oman (2008), Saudi Arabia (2008) and UAE (2009)(World Health Ogranization, 2013). The survey covered both the national and non-national adult populations in all four countries. Nationals and non-nationals experience healthcare differently in the GCC region need to be analysed separately. All surveys were nationally representative and conducted in person with full ethical permissions. In all surveys except Kuwait, where simple random sample was used, the survey design used 3-stage sample selection. Clusters (enumeration areas) were randomly selected with probability proportion to size (PPS) then a fixed sample of households were randomly selected from each cluster and eligible individuals were selected using Kish-tables. In Oman and Saudi the sample was stratified by place of residence and in UAE and Kuwait by nationality.

Three questions related to cervical and breast cancer screening were asked to eligible women during the individual interview:

1. When was the last time you had a pelvic examination, if ever?

2. The last time you had the pelvic examination, did you have a Pap smear test?

3. When was the last time you had a mammogram, if ever?

Further information to describe each procedure was also given if required for the respondent to identify if such an examination had occurred.

For the mammography analysis, the sample was restricted to women aged 40 to 75 years (for Oman the age range was 40 to 69 years due to those aged 70 or higher not being asked 
this question). Table 1 gives the number of respondents by country and nationality. The time period for rescreening was selected to be two years following WHO recommendations (World Health Organization, 2014). Response rates to screening questions were high in all countries apart from UAE.

---Table 1 about here---

Females between the ages of 25 and 64 years were questioned about uptake of cervical cancer screening through their use of a Pap smear. A Pap smear interval of three years has been selected for those aged between 25 and 49 years old, while a five-year interval is selected for women aged between 50 and 64, again following WHO recommendations (World Health Organization, 2013). The number of women in this age range for each country, and who provided a valid answer to the above questions, are shown in Table 1. A woman was only asked whether she had a Pap smear if she were to have already stated that she had a pelvic examination, and hence if the response to having an examination was negative, she was recorded as not having a Pap smear.

Women aged 40-64 are expected to have both mammograms and Pap smear tests. If these are combined the overall coverage of cancer screening amongst this group can be calculated. This is conducted for each country and for nationals and non-nationals separately. To ascertain whether women are receiving screening at longer intervals than are recommended the percentages of women aged 40-64 who received both examinations within the last 5 years was calculated, as well as within the last 10 years.

Household (highest educational level of males within the household, wealth, place of residence) and individual socio-demographic variables (age, marital status, education and employment) were selected for analysis as they were hypothesised to be associated with 
the uptake of regular screening. The highest education level among all male household members was used as a proxy for household influences on a woman's participation in screening. Wealth was calculated using principal components analysis on the assets held by the household to give a relative measure of wealth (Filmer and Pritchett, 2001), divided into tertiles of low, medium and high wealth. All results were calculated for national and nonnational populations separately where possible. Each country's questionnaires contained the same questions, except that Kuwait did not collect information about place of residence as the country is almost $100 \%$ urban.

\section{Analysis}

All three outcomes (Mammography, Pap smear and both together) were coded as binary indicating if the screen had occurred within the requisite period of time. Due to the small percentage of women who had a mammogram in required intervals, the analysis for this only focused on differences by age and nationality. The association between age and mammography use was tested using the Fisher's exact test. For Pap smear screening weighted cross-tabulations were produced between the screening and different background variables. The association between each background variables and mammography use was tested using the chi-square test.

In order to account for the different sampling design in the survey, all analyses applied in this study were weighted accordingly. Data analysis was performed using STATA version 13 (StataCorp, 2013). It was not possible to calculate confidence intervals for UAE as there was a single female respondent within a strata within the age range of interest. 


\section{Results}

\section{Mammography}

The levels of women who had a mammogram within the last two years was low in all countries and for both nationals and non-nationals. The lowest was in Saudi Arabia, with a weighted percentage of $4.9 \%$ (95\% $\mathrm{Cl}: 3.4 \%$ to $6.8 \%$ ), while the highest was in Kuwait, where almost $15 \%$ of women had been scanned ( $95 \% \mathrm{Cl}: 12.1 \%$ to $17.6 \%)$. In UAE only 33 women reported having a scan in the last two years out of a total sample size of 221 women (13.9\%), while in Oman there were only 32 women with a scan out of 742 women in the sample (8.9\%, $95 \% \mathrm{Cl}: 5.9 \%$ to $13.2 \%)$. Table 2 shows the percentage of women with a mammogram by nationality and age group. There is no clear and consistent evidence of differences by age group or nationality.

---Table 2 about here---

\section{Pap Smear Screening}

A higher percentage of women had a Pap smear in the recommended intervals than was seen for mammography. The percentage with a Pap smear ranged from $7.6 \%(95 \% \mathrm{Cl}: 6.4 \%$ to $9.0 \%$ ) in Saudi Arabia to $28.0 \%$ in the UAE. The corresponding percentages in Oman were $10.6 \%(95 \% \mathrm{Cl}: 8.6 \%$ to $13.0 \%)$ and $17.7 \%(95 \% \mathrm{Cl}: 15.6 \%$ to $20.0 \%)$ in Kuwait. The crosstabulations with background variables are shown in Table 3.

---Table 3 about here---

There were a number of significant associations between the background variables and the reporting of a Pap smear within recommended intervals. Nationality was significant in both Oman and UAE, although non-national women were more likely to be screened in Oman and nationals in UAE. Marital status was significant in all countries, with married 
women more likely to have been screened than those who were not married. There were also significant differences by place of residence in Oman, Saudi Arabia and UAE. Wealth was significantly related to cervical screening in all countries except Kuwait, although there was no consistent pattern across the countries regarding the direction of this relationship. Education was related to screening in both Oman and Saudi Arabia, with the most educated reporting the highest level of Pap screening.

\section{Overall Coverage}

Overall the percentage of women between ages 40 and 64 who have had both mammograms and Pap smears is extremely low, with only $2.2 \%$ of women in Saudi Arabia meeting the recommendations, rising to $6.3 \%$ in Kuwait (Table 4). Extending the intervals makes little difference to the coverage, with a 10 year reference period increasing the percentages of those screened to $2.7 \%$ for Saudi Arabia and $8.1 \%$ for Kuwait.

---Table 4 about here---

\section{Discussion}

The implementation of the WHS+ in the GCC region enables a wide range of comparative studies into different health outcomes in this region of the world. Comparing countries with similar backgrounds supports conclusions to be made about levels of health and health care in each country and the governmental policies that have been implemented. With the clear benefits of screening for both breast and cervical cancer for early diagnosis and treatment, alongside very limited national level data within the GCC countries, using the WHS+ to compare between these four countries should highlight good practice.

Rates of both mammography and screening for cervical cancer are extremely low in all countries under analysis. With a maximum percentage of women of relevant ages who have 
been screened of $14.6 \%$ for a mammogram (in Kuwait), and $28.0 \%$ for a Pap smear (in UAE), it is clear that all countries need to improve screening rates. If narrower age groups are used to align with up-to-date screening guidelines (World Health Organization, 2013, 2014) the rates of mammography screening only rise to a maximum of $25.2 \%$ in the UAE for $50-64$ year olds, and to $20.6 \%$ amongst $30-39$ year olds for Pap smears in Kuwait. Comparator countries outside of the GCC region have much higher levels of screening (Kobeissi et al., 2012 , OECD , 2015), indicating that there is scope for lessons to be learnt from countries with a higher screening rate through the adoption of new and updated policies.

There has been a debate about the efficacy of mammography at different ages, with a report concluding that the benefits of screening outweighing the risks of overtreatment for those aged 50-69 and 70-75 (Lauby-Secretan et al., 2015), with a limited reduction in risk seen for those aged 40-49. Yet the levels by age group for each of the countries indicate that women of all ages are screened at a far lower level than recommendations, leading to the conclusion that these low levels of screening are related to low levels of awareness of the availability of services and the potential benefits of the procedures. Clear requirements in each country for the frequency and ages where screening is beneficial would highlight best practice to women and may encourage greater participation in the programmes.

In the four countries under analysis here national health awareness campaigns to increase the rates of screening have been sporadic, especially at the time the data was collected. A study in Saudi Arabia indicated that the national breast cancer screening centre, established in 2007, was only effectively accessed by women from Riyadh, where it was based (Alhazmi, 2016). Further campaigns have been noted in all countries, especially focusing on mobile clinics, but the effectiveness of these to increase screening rates amongst women is unknown. In 2007 the Gulf Plan for Cancer Control emphasized that 
there was a need for greater awareness of the risk factors for all cancers, and there has been further efforts to increase screening rates in all GCC countries, although the effectiveness of these efforts is currently unknown.

To encourage women to use screening programmes the underlying reasons why there was low uptake need to be investigated further. This study has indicated that there is a significant relationship between cervical cancer screening and marital status in all countries, with wealth, the level of education of the woman, place of residence and nationality also related in some countries. These are unadjusted relationships, so there may be some confounding that is not accounted for. Some of these relationships are simple to explain, with those from rural areas having poorer access to health facilities, while higher educated women are likely to be more informed about the benefits of screening and greater autonomy to attend. The relationship between marital status and Pap screens may be partly due to age, but there are known issues with unmarried women using the health system within the GCC region (Mobaraki and Söderfeldt, 2010). Further research looking at the barriers to screening will elucidate this relationship further.

This study only indicates the association of screening with a range of factors and cannot elucidate the underlying factors further. However, studies in the region have indicated a lack of knowledge about cancer and screening (Al-Meer et al., 2011), a lack of support from husbands (El Saghir et al., 2007) and from health providers (Bener et al., 2001, Al Sairafi and Mohamed, 2009), as well as fear of the process (Amin et al., 2009). A recent study into cervical cancer knowledge in Oman highlighted that specific knowledge of the signs and symptoms of the cancer, alongside that of the Pap smear itself, was low, especially amongst the least educated (Nasar et al., 2016).

\section{Limitations}


The estimates of women who have been screened are based on self-reported screening, which could suffer from reporting bias. For example, it is possible that the percentages of women who have Pap smears is higher than those recorded in the survey, with women not reporting for a number of reasons, such as that they were not formally told that a procedure was called a Pap smear when it was conducted. The question relating to Pap smears was only asked if a pelvic examination was conducted, which may have been misinterpreted and thus not reported. A further issue was with the high proportion of missing data in UAE, with almost half of the eligible women not answering questions about screening leading to limits to the generalizability of the results.. However, in the three other countries there was a low level of missing data, especially in Saudi Arabia and Oman. Finally, the data were collected between 2008 and 2009 and hence they may not reflect the current situation. Yet these data allow the establishment of a good baseline from which progress can be judged.

\section{Conclusions}

Levels of cancer screening in the GCC countries analysed are low. Even using the most liberal definition of a 10 year window for screening, less than $10 \%$ of women aged 40 to 64 had both a mammogram and Pap smear. Although there are other tests that can be used for breast and cervical cancer identification, these low levels of the common screening tests may lead to a higher level of undetected cancers amongst women, with the higher mortality that will result. This is within the context of an ageing population and high noncommunicable disease burden.

This study has shown that across Kuwait, Oman, Saudi Arabia and UAE there is a need for an increased awareness of cancer screening in the population. There needs to be a specific focus on expanding screening for both cervical and breast cancer to encourage women to 
use the facilities that are available. Inequalities in use between population groups are observed, but with the low levels that are observed a key requirement is to improve utilisation in all groups through targeted public education campaigns in all four GCC countries, through a united action that brings together these countries. 


\section{References}

Al-Amoudi SM, Abdulijabbar HS (2012). Men's knowledge and attitude towards breast cancer in Saudi Arabia. Saudi Medical Journal 33(5):547-550.

Al-Meer F, Aseel M, Al-Khalaf J, Al-Kuwari M, Ismail M (2011). Knowledge, attitude and practices regarding cervical cancer and screening among women visiting primary health care in Qatar. Eastern Mediterranean health journal 17(11):855.

Al-Othman S, Haoudi A, Alhomoud S, Alkhenizan A, Khoja T, Al-Zahrani A (2015). Tackling Cancer Control in the Gulf Cooperation Council Countries. The Lancet Oncology 16(5):246-257. doi: 10.1016/s1470-2045(15)70034-3.

Al Eyd GJ, Shaik RB (2012). Rate of Opportunistic Pap Smear Screening and Patterns of Epithelial Cell Abnormalities in Pap Smears in Ajman, United Arab Emirates. Sultan Qaboos University Medical Journal 12(4):473-478.

Al Sairafi M, Mohamed FA (2009). Knowledge, Attitudes, and Practice Related to Cervical Cancer Screening among Kuwaiti Women. Medical Principles and Practice 18(1):35-42.

Alhazmi FG (2016). Comparison of Breast and Colorectal Cancer Screening Programs in the Netherlands and the Kingdom of Saudi Arabia. Cancer 4(1):157-165.

Amin TT, Al Mulhim A, Al Meqihwi A (2009). Breast cancer knowledge, risk factors and screening among adult Saudi women in a primary health care setting. Asian Pac J Cancer Prev 10(1):133-138.

Bener A, Alwash R, Miller CJ, Denic S, Dunn EV (2001). Knowledge, attitudes, and practices related to breast cancer screening: A survey of Arabic women. Journal of Cancer Education 16(4):215-220. doi: 10.1080/08858190109528776.

Bener A, El Ayoubi H, Moore M, Basha B, Joseph S, Chouchane L (2009). Do we need to maximise the breast cancer screening awareness? Experience with an endogamous society with high fertility. Asian Pacific Journal of Cancer Prevention 10(4):1-6. 
Donnelly TT, Al Khater A-H, Al-Bader SB, Al Kuwari MG, Malik M, Al-Meer N et al. (2015a). Factors that Influence Awareness of Breast Cancer Screening among Arab Women in Qatar: Results from a Cross Sectional Survey. Asian Pacific Journal of Cancer Prevention 15(23):10157-10164. doi: 10.7314/apjcp.2014.15.23.10157.

Donnelly TT, Al Khater A-H, Al-Bader SB, Al Kuwari MG, Al-Meer N, Malik M et al. (2012). Breast cancer screening among Arabic women living in the State of Qatar: Awareness, knowledge, and participation in screening activities. Avicenna (2012):2. doi: 10.5339/avi.2012.2.

Donnelly TT, Al Khater AH, Al Kuwari MG, Al-Bader SB, Al-Meer N, Abdulmalik M et al. (2015b). Do socioeconomic factors influence breast cancer screening practices among Arab women in Qatar? BMJ open 5(1):e005596. doi: 10.1136/bmjopen-2014-005596.

El Bcheraoui C, Basulaiman M, Wilson S, Daoud F, Tuffaha M, AlMazroa MA et al. (2015). Breast Cancer Screening in Saudi Arabia: Free but Almost No Takers. PLOS ONE 10(3):e0119051. doi: 10.1371/journal.pone.0119051.

El Saghir NS, Khalil MK, Eid T, El Kinge AR, Charafeddine M, Geara F et al. (2007). Trends in epidemiology and management of breast cancer in developing Arab countries: a literature and registry analysis. International journal of surgery 5(4):225-233.

Ervik M, Lam F, Ferlay J, Mery I, Soerjomataram I, Bray F. Cancer Today. Cancer Today. Lyon, France: International Agency for Research on Cancer; 2016.

Ferlay J, Soerjomataram I, Dikshit R, Eser S, Mathers C, Rebelo M et al. (2015). Cancer incidence and mortality worldwide: Sources, methods and major patterns in GLOBOCAN 2012. International Journal of Cancer 136(5):E359-E386. doi: 10.1002/ijc.29210.

Filmer D, Pritchett L (2001). Estimating Wealth Effects Without Expenditure Data-Or Tears: An Application To Educational Enrollments In States Of India*. Demography 38(1):115-132. doi: 10.1353/dem.2001.0003. 
Kobeissi L, Hamra R, Samari G, Koleilat L, Khalifeh M (2012). The 2009 Lebanese National Mammography Campaign: Results and Assessment Using a Survey Design. Epidemiology: Open Access 2(112).

Latif R (2014). Knowledge and attitude of Saudi female students towards breast cancer: A cross-sectional study. Journal of Taibah University Medical Sciences 9(4):328-334. doi:

10.1016/j.jtumed.2014.05.004.

Lauby-Secretan B, Scoccianti C, Loomis D, Benbrahim-Tallaa L, Bouvard V, Bianchini F et al. (2015). Breast-Cancer Screening - Viewpoint of the IARC Working Group. New England Journal of Medicine 372(24):2353-2358. doi: doi:10.1056/NEJMsr1504363.

Mobaraki A, Söderfeldt B (2010). Gender inequity in Saudi Arabia and its role in public health/L'inégalité entre hommes et femmes en Arabie saoudite et ses conséquences sur la santé publique. Eastern Mediterranean health journal 16(1):113.

Nasar A, Waad A, Atheer A, Nasra A (2016). Awareness of Cervical Cancer and Pap Smear Testing Among Omani Women. Asian Pacific journal of cancer prevention: APJCP 17(11):4825-4830.

OECD. Health at a Glance 2015: OECD Indicators. OECD Publishing, Paris2015.

Ravichandran K, Al-Hamdan NA, Mohamed G (2011). Knowledge, attitude, and behavior among Saudis toward cancer preventive practice. Journal of family \& community medicine 18(3):135-142. doi: 10.4103/2230-8229.90013.

Sait K, Bentley J, Anfinan N, Power P (2012). Cervical Cancer Prevention in Saudi Arabia: It is Time to Call for Action! The Open Women's Health Journal 6:1-5.

StataCorp. STATA Statistical Software: Release 13. College Station, TX: StataCorp LP.; 2013. World Health Ogranization. WHO Study on global AGEing and adult health (SAGE). 2013. World Health Organization. WHO guidelines for screening and treatment of precancerous lesions for cervical cancer prevention. Geneva, Switzerland2013.

World Health Organization. WHO Position Paper on Mammography Screening. 2014. World Health Organization. Cancer. Fact Sheet No 2972015. 
Table 1: Analytic sample size for mammography and Pap smear screening, by country

\begin{tabular}{|c|c|c|c|c|}
\hline & Kuwait & Oman & Saudi & UAE \\
\hline Total households in survey & 4389 & 5465 & 8988 & 3422 \\
\hline Total individuals in survey & 3828 & 4717 & 8329 & 2579 \\
\hline \multicolumn{5}{|l|}{ Mammography } \\
\hline Total females aged $40-75$ in sample & 851 & $743^{+}$ & 1223 & 453 \\
\hline Total respondents & 697 & 742 & 1211 & 221 \\
\hline Response rate & $81.9 \%$ & $99.9 \%$ & $99.0 \%$ & $48.8 \%$ \\
\hline \multicolumn{5}{|l|}{ Nationality } \\
\hline National & 555 & 664 & 964 & 133 \\
\hline Non-national & 142 & 78 & 247 & 88 \\
\hline \multicolumn{5}{|l|}{ Pap smear screening } \\
\hline Total females aged $25-64$ in sample & 1695 & 1657 & 2781 & 1128 \\
\hline Total respondents & 1526 & 1655 & 2746 & 533 \\
\hline Response rate & $90.0 \%$ & $99.9 \%$ & $98.7 \%$ & $47.3 \%$ \\
\hline \multicolumn{5}{|l|}{ Nationality } \\
\hline National & 1115 & 1457 & 2107 & 236 \\
\hline Non-national & 411 & 198 & 639 & 297 \\
\hline
\end{tabular}

*All figures are unweighted.

+ The age range for Oman was 40-69 
Table 2: Percentage of women who received a mammogram in the two years prior to the survey, by nationality and age, for Kuwait, Oman,

\section{Saudi Arabia and UAE}

\begin{tabular}{|c|c|c|c|c|c|c|c|c|c|c|c|c|c|c|c|c|c|}
\hline & \multirow[b]{2}{*}{ Age } & \multicolumn{4}{|c|}{ Kuwait } & \multicolumn{4}{|c|}{ Oman } & \multicolumn{4}{|c|}{ Saudi Arabia } & \multicolumn{4}{|c|}{ UAE } \\
\hline & & & Had & & $\mathrm{p}-$ & & Had & & $\mathrm{p}-$ & & Had & & $\mathrm{p}-$ & & Had & & $\mathrm{p}-$ \\
\hline & group & $\mathrm{n}$ & scan & $\%$ & value* & $\mathrm{n}$ & scan & $\%$ & value* & $\mathrm{n}$ & scan & $\%$ & value* & $\mathrm{n}$ & scan & $\%$ & value* \\
\hline \multirow[t]{4}{*}{$\underline{\text { Nationals }}$} & All ages & 555 & 93 & $15.0 \%$ & 0.083 & 664 & 19 & $5.6 \%$ & 0.173 & 964 & 32 & $4.2 \%$ & 0.194 & 133 & 20 & $15.4 \%$ & 0.247 \\
\hline & $40-49$ & 248 & 32 & $11.0 \%$ & & 262 & 9 & $5.0 \%$ & & 465 & 12 & $3.2 \%$ & & 67 & 11 & $14.0 \%$ & \\
\hline & $50-64$ & 238 & 48 & $17.9 \%$ & & 304 & 10 & $7.9 \%$ & & 360 & 17 & $5.9 \%$ & & 52 & 9 & $21.3 \%$ & \\
\hline & $65-75$ & 69 & 13 & $16.6 \%$ & & 98 & 0 & $0.0 \%$ & & 139 & 3 & $3.1 \%$ & & 14 & 0 & $0.0 \%$ & \\
\hline \multirow[t]{3}{*}{ Non-nationals } & All ages & 142 & 23 & $13.1 \%$ & 0.168 & 78 & 13 & $23.0 \%$ & 0.435 & 247 & 14 & $7.0 \%$ & 0.100 & 88 & 13 & $12.3 \%$ & 0.438 \\
\hline & $40-49$ & 95 & 12 & $11.7 \%$ & & 54 & 11 & $30.0 \%$ & & 149 & 10 & $8.2 \%$ & & 63 & 8 & $7.4 \%$ & \\
\hline & $50-64$ & 44 & 11 & $17.7 \%$ & & 23 & 2 & $4.2 \%$ & & 84 & 2 & $3.4 \%$ & & 21 & 4 & $34.9 \%$ & \\
\hline \multirow[t]{4}{*}{ Total } & All ages & 697 & 116 & $14.6 \%$ & 0.023 & 742 & 32 & $8.9 \%$ & 0.010 & 1211 & 46 & $4.9 \%$ & 0.850 & 221 & 33 & $13.9 \%$ & 0.523 \\
\hline & $40-49$ & 343 & 44 & $11.2 \%$ & & 316 & 20 & $13.2 \%$ & & 614 & 22 & $4.5 \%$ & & 130 & 19 & $10.1 \%$ & \\
\hline & $50-64$ & 282 & 59 & $17.9 \%$ & & 327 & 12 & $7.5 \%$ & & 444 & 19 & $5.4 \%$ & & 73 & 13 & $25.2 \%$ & \\
\hline & $65-75$ & 72 & 13 & $15.0 \%$ & & 99 & 0 & $0.0 \%$ & & 153 & 5 & $4.6 \%$ & & 18 & 1 & $7.1 \%$ & \\
\hline
\end{tabular}

* Fishers exact test was used 
Table 3: Percentage of women who received a Pap smear within recommended intervals ${ }^{\dagger}$ prior to the survey by selected co-variates, for Kuwait, Oman, Saudi Arabia and UAE

\begin{tabular}{|c|c|c|c|c|c|c|c|c|c|c|c|c|c|}
\hline & & \multicolumn{3}{|c|}{ Kuwait } & \multicolumn{3}{|c|}{ Oman } & \multicolumn{3}{|c|}{ Saudi Arabia } & \multicolumn{3}{|c|}{ UAE } \\
\hline & & Had Pap & & & Had Pap & & & Had Pap & & & Had Pap & & \\
\hline & & Test & $\%$ & $p$-value* & Test & $\%$ & $\mathrm{p}$-value* & Test & $\%$ & p-value* & Test & $\%$ & $p$-value* \\
\hline \multirow[t]{3}{*}{ Total } & & 292 & $17.7 \%$ & & 133 & $10.6 \%$ & & 194 & $7.6 \%$ & & 139 & $28.0 \%$ & \\
\hline & $25-29$ years old & 43 & $14.2 \%$ & 0.203 & 31 & $7.8 \%$ & 0.525 & 30 & $5.4 \%$ & 0.006 & 19 & $20.7 \%$ & 0.320 \\
\hline & 30-39 years old & 113 & $20.6 \%$ & & 59 & $12.4 \%$ & & 97 & $9.2 \%$ & & 63 & $27.5 \%$ & \\
\hline \multicolumn{14}{|l|}{ Age group } \\
\hline & $40-49$ years old & 80 & $18.8 \%$ & & 25 & $11.4 \%$ & & 51 & $9.2 \%$ & & 42 & $34.7 \%$ & \\
\hline & $50-64$ years old & 56 & $16.1 \%$ & & 18 & $9.8 \%$ & & 16 & $4.3 \%$ & & 15 & $32.3 \%$ & \\
\hline & National & 223 & $18.7 \%$ & 0.118 & 103 & $8.4 \%$ & 0.002 & 151 & $7.8 \%$ & 0.480 & 72 & $33.2 \%$ & 0.046 \\
\hline & Non-national & 69 & $15.0 \%$ & & 30 & $18.1 \%$ & & 43 & $6.9 \%$ & & 67 & $23.3 \%$ & \\
\hline \multirow{2}{*}{\multicolumn{14}{|c|}{ Place of Residence }} \\
\hline & & & & & & & & & & & & & \\
\hline & Rural & NA & - & & 42 & $6.3 \%$ & & 23 & $4.6 \%$ & & 96 & $38.0 \%$ & \\
\hline \multirow{3}{*}{ Marital statu } & Not Married & 24 & $7.1 \%$ & $<0.001$ & 8 & $4.0 \%$ & 0.010 & 13 & $3.0 \%$ & $<0.001$ & 13 & $16.1 \%$ & 0.043 \\
\hline & Married & 268 & $20.5 \%$ & & 125 & $12.4 \%$ & & 181 & $8.5 \%$ & & 126 & $30.4 \%$ & \\
\hline & Low (Primary or less) & 31 & $15.0 \%$ & 0.268 & 58 & $6.5 \%$ & 0.006 & 78 & $6.4 \%$ & 0.041 & 28 & $25.5 \%$ & 0.829 \\
\hline \multirow[t]{2}{*}{ Education } & Medium (Secondary) & 144 & $19.6 \%$ & & 47 & $13.4 \%$ & & 58 & $7.5 \%$ & & 50 & $29.7 \%$ & \\
\hline & High (College or above) & 117 & $16.4 \%$ & & 28 & $15.4 \%$ & & 58 & $10.0 \%$ & & 61 & $27.9 \%$ & \\
\hline Employment & Employed & 126 & $17.1 \%$ & 0.911 & 23 & $14.0 \%$ & 0.216 & 32 & $7.5 \%$ & 0.996 & 26 & $29.2 \%$ & 0.025 \\
\hline
\end{tabular}




\begin{tabular}{|c|c|c|c|c|c|c|c|c|c|c|c|c|c|}
\hline & Unemployed & 48 & $18.5 \%$ & & 13 & $6.6 \%$ & & 44 & $7.6 \%$ & & 14 & $11.9 \%$ & \\
\hline & Homemaker/ retired & 117 & $17.8 \%$ & & 97 & $10.7 \%$ & & 118 & $7.6 \%$ & & 99 & $31.8 \%$ & \\
\hline Highest educational & Low (Primary or less) & 106 & $19.2 \%$ & 0.334 & 37 & $10.2 \%$ & 0.840 & 60 & $5.7 \%$ & 0.152 & 28 & $29.2 \%$ & 0.031 \\
\hline level of a male in the & Medium (Secondary) & 86 & $15.6 \%$ & & 2 & $7.4 \%$ & & 70 & $8.1 \%$ & & 35 & $17.7 \%$ & \\
\hline \multirow[t]{2}{*}{ household } & High (College or above) & 47 & $19.0 \%$ & & 68 & $10.8 \%$ & & 64 & $9.6 \%$ & & 76 & $32.8 \%$ & \\
\hline & Low & 70 & $14.5 \%$ & 0.221 & 34 & $9.3 \%$ & 0.024 & 21 & $2.5 \%$ & $<0.001$ & 61 & $39.9 \%$ & $<0.001$ \\
\hline \multirow[t]{2}{*}{ Wealth } & Medium & 119 & $18.7 \%$ & & 31 & $6.5 \%$ & & 76 & $7.5 \%$ & & 31 & $17.3 \%$ & \\
\hline & High & 103 & $18.9 \%$ & & 68 & $13.1 \%$ & & 97 & $10.1 \%$ & & 47 & $28.7 \%$ & \\
\hline
\end{tabular}


Table 4: Percentage of women aged 40-64 who have received both mammography and Pap smear screening for different reference periods

\begin{tabular}{|c|c|c|c|c|c|c|c|c|}
\hline & \multicolumn{2}{|c|}{ Kuwait } & \multicolumn{2}{|c|}{ Oman } & \multicolumn{2}{|c|}{ Saudi Arabia } & \multicolumn{2}{|c|}{ UAE } \\
\hline & $\mathrm{n}$ & $\%$ & $\mathrm{n}$ & $\%$ & $\mathrm{n}$ & $\%$ & $\mathrm{n}$ & $\%$ \\
\hline Count of women aged $40-64$ & 768 & & 644 & & 1067 & & 418 & \\
\hline Count of respondents to both questions & 694 & $90.4 \%$ & 643 & $99.8 \%$ & 1057 & $99.1 \%$ & 224 & $53.6 \%$ \\
\hline \multicolumn{9}{|l|}{ National } \\
\hline \multicolumn{9}{|l|}{ Both screenings within : } \\
\hline \multicolumn{9}{|l|}{ Recommendations* } \\
\hline Last 5 years & 51 & $7.6 \%$ & 10 & $3.3 \%$ & 12 & $2.0 \%$ & 17 & $7.4 \%$ \\
\hline Last 10 years & 54 & $8.0 \%$ & 10 & $3.3 \%$ & 14 & $2.3 \%$ & 18 & $7.9 \%$ \\
\hline
\end{tabular}

\section{Non-national}

Both screenings within:

$\begin{array}{lcccccccc} & 13 & 6.2 \% & 7 & 11.7 \% & 5 & 3.1 \% & 7 & 3.5 \% \\ \text { Recommendations* } & & & & & & & & \\ \text { Last } 5 \text { years } & 15 & 7.3 \% & 7 & 11.7 \% & 7 & 4.0 \% & 7 & 3.5 \% \\ \text { Last } 10 \text { years } & 16 & 8.3 \% & 8 & 14.8 \% & 7 & 4.0 \% & 8 & 3.6 \%\end{array}$

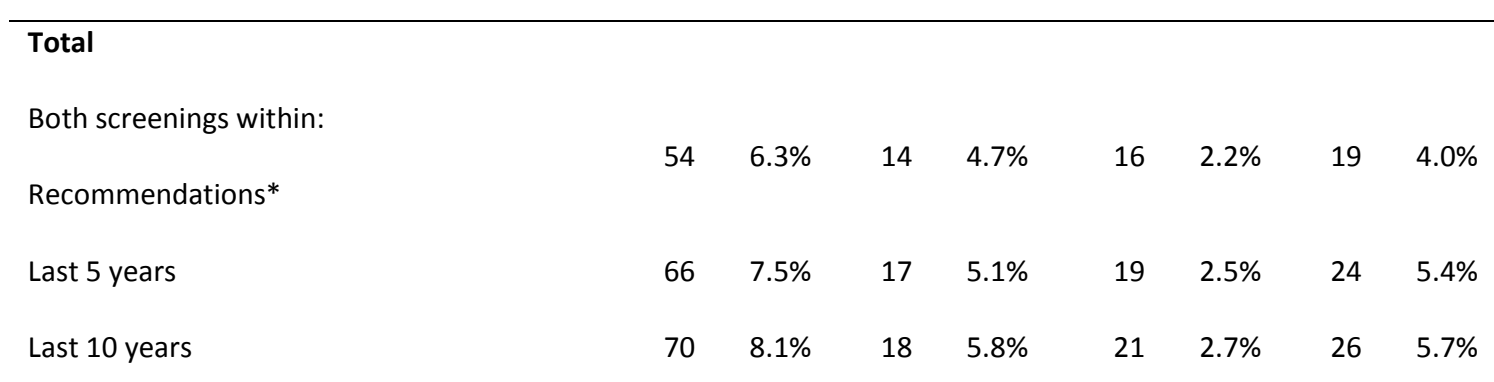

* Mammography within two years, Pap smear within three years for those aged 40-49 years and within five years for those aged $50-64$ years 